\title{
Correction to: Spatio-temporal trends in monthly pan evaporation in Aguascalientes, Mexico
}

\author{
Osias Ruiz-Alvarez ${ }^{1,2} \cdot$ Vijay P. Singh ${ }^{3}$. Juan Enciso-Medina ${ }^{4} \cdot$ Clyde Munster $^{3} \cdot$ Ronald Kaiser $^{1}$. \\ Ronald Ernesto Ontiveros-Capurata ${ }^{5} \cdot$ Luis Antonio Diaz-Garcia $^{2} \cdot$ Carlos Antonio Costa dos Santos $^{6}$
}

Published online: 7 June 2018

(C) Springer-Verlag GmbH Austria, part of Springer Nature 2018

\section{Correction to: Theoretical and Applied Climatology https://doi.org/10.1007/s00704-018-2491-8}

The original version of this article unfortunately contained a mistake. Tables 1 and 2 have errors. The presentation of Table 2 was incorrect was not fixed even after being pointed out in the proofs. Furthermore, the information of Table 2 is copied in Table 1. The corrected Tables 1 and 2 are given below. Also, the 3rd author's name and the 5th institution have been corrected.

The original article has been corrected.

The online version of the original article can be found at https://doi.org/ $10.1007 / \mathrm{s} 00704-018-2491-8$

Osias Ruiz-Alvarez

oruizalvarez454483@tamu.edu; ruiz.osias@inifap.gob.mx

1 Water Management and Hydrological Science, Texas A\&M

University, College Station, TX 77843, USA

2 Instituto Nacional de Investigaciones Forestales Agrícolas y Pecuarias (INIFAP), 20660 Pabellón de Arteaga, Aguascalientes, Mexico

3 Department of Biological and Agricultural Engineering, Texas A\&M University, College Station, TX 77843, USA

4 Department of Biological and Agricultural Engineering, Texas A\&M AgriLife Research, Weslaco, TX 78596, USA

5 CONACYT-Instituto Mexicano de Tecnología del Agua (IMTA), 62550 Jiutepec, Morelos, Mexico

6 Department of Atmospheric Science, Federal University of Campina Grande, Avenida Aprígio Veloso, 882, Bodocongó, Campina Grande, Paraiba 58109-970, Brazil 
Table 1 Characteristics of 52 weather stations used in the study

\begin{tabular}{|c|c|c|c|c|c|}
\hline No. & Weather station & Latitude $(\mathrm{N})$ & Longitude (W) & Elevation (m) & Duration of data \\
\hline 1 & Cañada Honda & $22^{\circ} 00^{\prime} 0^{\prime \prime}$ & $102^{\circ} 11^{\prime} 56^{\prime \prime}$ & 1702 & $1970-2008$ \\
\hline 2 & Presa El Niagara & $21^{\circ} 46^{\prime} 44^{\prime \prime}$ & $102^{\circ} 22^{\prime} 19^{\prime \prime}$ & 1828 & $1957-2008$ \\
\hline 3 & Jesus Maria & $21^{\circ} 57^{\prime} 0^{\prime \prime}$ & $102^{\circ} 21^{\prime} 0^{\prime \prime}$ & 1800 & 1949-1988 \\
\hline 4 & Puerto De La Concepcion & $22^{\circ} 12^{\prime} 7^{\prime \prime}$ & $102^{\circ} 08^{\prime} 6^{\prime \prime}$ & 2300 & $1973-2008$ \\
\hline 5 & La Tinaja & $22^{\circ} 09^{\prime} 50^{\prime \prime}$ & $102^{\circ} 33^{\prime} 14^{\prime \prime}$ & 2425 & $1971-2008$ \\
\hline 6 & Malpaso & $21^{\circ} 51^{\prime} 36^{\prime \prime}$ & $102^{\circ} 39^{\prime} 50^{\prime \prime}$ & 1775 & $1958-2008$ \\
\hline 7 & Presa Media Luna & $21^{\circ} 47^{\prime} 38^{\prime \prime}$ & $102^{\circ} 48^{\prime} 7^{\prime \prime}$ & 1585 & $1970-2008$ \\
\hline 8 & Mesillas & $22^{\circ} 18^{\prime} 47^{\prime \prime}$ & $102^{\circ} 09^{\prime} 58^{\prime \prime}$ & 1990 & $1963-2008$ \\
\hline 9 & Pabellon Campo Experimental & $22^{\circ} 10^{\prime} 1^{\prime \prime}$ & $102^{\circ} 17^{\prime} 35^{\prime \prime}$ & 1909 & 1946-2006 \\
\hline 10 & Palo Alto & $21^{\circ} 54^{\prime} 58^{\prime \prime}$ & $101^{\circ} 58^{\prime} 8^{\prime \prime}$ & 2015 & $1970-2008$ \\
\hline 11 & Ganaderia Peñuelas & $21^{\circ} 42^{\prime} 0^{\prime \prime}$ & $102^{\circ} 18^{\prime} 0^{\prime \prime}$ & 1878 & $1945-1970$ \\
\hline 12 & Presa Potrerillos & $22^{\circ} 13^{\prime} 59^{\prime \prime}$ & $102^{\circ} 26^{\prime} 38^{\prime \prime}$ & 2090 & $1947-2008$ \\
\hline 13 & Presa Plutarco Elias Calles & $22^{\circ} 08^{\prime} 28^{\prime \prime}$ & $102^{\circ} 24^{\prime} 54^{\prime \prime}$ & 2020 & $1937-2008$ \\
\hline 14 & Presa Jocoque & $22^{\circ} 07^{\prime} 41^{\prime \prime}$ & $102^{\circ} 21^{\prime} 32^{\prime \prime}$ & 1970 & $1942-2008$ \\
\hline 15 & Presa La Codorniz & $21^{\circ} 59^{\prime} 49^{\prime \prime}$ & $102^{\circ} 40^{\prime} 26^{\prime \prime}$ & 1783 & 1963-2008 \\
\hline 16 & San Bartolo & $21^{\circ} 44^{\prime} 53^{\prime \prime}$ & $102^{\circ} 10^{\prime} 12^{\prime \prime}$ & 1965 & $1949-2000$ \\
\hline 17 & Calvillo & $21^{\circ} 50^{\prime} 13^{\prime \prime}$ & $102^{\circ} 42^{\prime} 43^{\prime \prime}$ & 1665 & 1959-2008 \\
\hline 18 & San Isidro & $21^{\circ} 46^{\prime} 40.80^{\prime \prime}$ & $102^{\circ} 5^{\prime} 14.40^{\prime \prime}$ & 1895 & $1970-2008$ \\
\hline 19 & Tepezala & $22^{\circ} 13^{\prime} 23^{\prime \prime}$ & $102^{\circ} 10^{\prime} 8^{\prime \prime}$ & 2110 & $1962-2008$ \\
\hline 20 & Venadero & $21^{\circ} 52^{\prime} 37^{\prime \prime}$ & $102^{\circ} 27^{\prime} 47^{\prime \prime}$ & 1995 & $1951-2008$ \\
\hline 21 & Villa Juarez & $22^{\circ} 06^{\prime} 4^{\prime \prime}$ & $102^{\circ} 04^{\prime} 5^{\prime \prime}$ & 1970 & $1958-2003$ \\
\hline 22 & Asientos & $22^{\circ} 14^{\prime} 31^{\prime \prime}$ & $102^{\circ} 25^{\prime} 12^{\prime \prime}$ & 2155 & 1963-2008 \\
\hline 23 & Aguascalientes & $21^{\circ} 53^{\prime} 42^{\prime \prime}$ & $102^{\circ} 18^{\prime} 29^{\prime \prime}$ & 1865 & $1947-2008$ \\
\hline 24 & El Novillo & $22^{\circ} 01^{\prime} 8^{\prime \prime}$ & $101^{\circ} 59^{\prime} 56^{\prime \prime}$ & 2010 & $1972-2008$ \\
\hline 25 & Las Fraguas & $22^{\circ} 02^{\prime} 20^{\prime \prime}$ & $101^{\circ} 53^{\prime} 31^{\prime \prime}$ & 2020 & $1972-2008$ \\
\hline 26 & Los Conos & $21^{\circ} 53^{\prime} 49^{\prime \prime}$ & $101^{\circ} 59^{\prime} 31^{\prime \prime}$ & 2015 & $1972-2008$ \\
\hline 27 & Sandovales & $21^{\circ} 53^{\prime} 6^{\prime \prime}$ & $102^{\circ} 06^{\prime} 32^{\prime \prime}$ & 2000 & $1972-2008$ \\
\hline 28 & Agua Zarca & $21^{\circ} 58^{\prime} 26^{\prime \prime}$ & $102^{\circ} 35^{\prime} 2^{\prime \prime}$ & 2300 & 1976-2008 \\
\hline 29 & Arellano & $21^{\circ} 48^{\prime} 7^{\prime \prime}$ & $102^{\circ} 16^{\prime} 23^{\prime \prime}$ & 1890 & 1979-2008 \\
\hline 30 & La Tinaja II & $21^{\circ} 48^{\prime} 36^{\prime \prime}$ & $102^{\circ} 07^{\prime} 44^{\prime \prime}$ & 2010 & 1977-2008 \\
\hline 31 & Cieneguilla & $21^{\circ} 43^{\prime} 52^{\prime \prime}$ & $102^{\circ} 27^{\prime} 11^{\prime \prime}$ & 1780 & 1979-2008 \\
\hline 32 & Montoro & $21^{\circ} 45^{\prime} 25^{\prime \prime}$ & $102^{\circ} 18^{\prime} 7^{\prime \prime}$ & 1855 & 1979-2008 \\
\hline 33 & Los Negritos & $21^{\circ} 52^{\prime} 12^{\prime \prime}$ & $102^{\circ} 20^{\prime} 56^{\prime \prime}$ & 1845 & 1980-2008 \\
\hline 34 & El Ocote I & $21^{\circ} 46^{\prime} 55^{\prime \prime}$ & $102^{\circ} 31^{\prime} 1^{\prime \prime}$ & 2005 & 1979-2008 \\
\hline 35 & El Ocote II & $21^{\circ} 53^{\prime} 24^{\prime \prime}$ & $102^{\circ} 49^{\prime} 55^{\prime \prime}$ & 2275 & $1980-2008$ \\
\hline 36 & Peñuelas & $21^{\circ} 43^{\prime} 34^{\prime \prime}$ & $102^{\circ} 16^{\prime} 19^{\prime \prime}$ & 1860 & 1979-2008 \\
\hline 37 & Presa Canutillo & $21^{\circ} 50^{\prime} 13^{\prime \prime}$ & $102^{\circ} 31^{\prime} 19^{\prime \prime}$ & 1930 & 1979-2008 \\
\hline 38 & Rancho Seco & $22^{\circ} 05^{\prime} 17^{\prime \prime}$ & $101^{\circ} 58^{\prime} 1^{\prime \prime}$ & 2055 & 1979-2008 \\
\hline 39 & Rincon De Romos & $22^{\circ} 13^{\prime} 52^{\prime \prime}$ & $102^{\circ} 18^{\prime} 54^{\prime \prime}$ & 1947 & 1979-2008 \\
\hline 40 & San Francisco de Los Romo & $22^{\circ} 04^{\prime} 44^{\prime \prime}$ & $102^{\circ} 16^{\prime} 23^{\prime \prime}$ & 1885 & 1979-2008 \\
\hline 41 & San Gil & $22^{\circ} 12^{\prime} 29^{\prime \prime}$ & $102^{\circ} 01^{\prime} 19^{\prime \prime}$ & 2010 & 1979-2008 \\
\hline 42 & Tepetatillo & $22^{\circ} 05^{\prime} 28^{\prime \prime}$ & $102^{\circ} 10^{\prime} 52^{\prime \prime}$ & 2020 & 1979-2008 \\
\hline 43 & Cosio & $22^{\circ} 21^{\prime} 47^{\prime \prime}$ & $102^{\circ} 17^{\prime} 49^{\prime \prime}$ & 1885 & 1978-2008 \\
\hline 44 & Presa 50 aniversario & $22^{\circ} 11^{\prime} 20^{\prime \prime}$ & $102^{\circ} 27^{\prime} 50^{\prime \prime}$ & 2050 & $1980-2004$ \\
\hline 45 & Jesus Maria, Jesus Maria & $21^{\circ} 57^{\prime} 11^{\prime \prime}$ & $102^{\circ} 20^{\prime} 31^{\prime \prime}$ & 1907 & 1979-2008 \\
\hline 46 & Los Alisos & $21^{\circ} 44^{\prime} 31^{\prime \prime}$ & $102^{\circ} 42^{\prime} 58^{\prime \prime}$ & 2040 & 1981-2008 \\
\hline 47 & El Chayote & $22^{\circ} 17^{\prime} 10^{\prime \prime}$ & $102^{\circ} 14^{\prime} 10^{\prime \prime}$ & 1930 & 1983-2008 \\
\hline 48 & Milpillas De Arriba & $21^{\circ} 56^{\prime} 6^{\prime \prime}$ & $102^{\circ} 33^{\prime} 4^{\prime \prime}$ & 2140 & 1982-2008 \\
\hline 49 & Calvillito & $21^{\circ} 50^{\prime} 6^{\prime \prime}$ & $102^{\circ} 10^{\prime} 55^{\prime \prime}$ & 1950 & 1983-2008 \\
\hline 50 & La Posta UAA & $21^{\circ} 58^{\prime} 19.20^{\prime \prime}$ & $102^{\circ} 21^{\prime} 43.20^{\prime \prime}$ & 1905 & $1986-2008$ \\
\hline 51 & Jesus Teran (El Muerto) & $21^{\circ} 58^{\prime} 34^{\prime \prime}$ & $102^{\circ} 03^{\prime} 43^{\prime \prime}$ & 2040 & 1985-2008 \\
\hline 52 & Las Presas, El Llano & $21^{\circ} 54^{\prime} 32.40^{\prime \prime}$ & $102^{\circ} 5^{\prime} 13.20^{\prime \prime}$ & 2025 & 1986-2008 \\
\hline
\end{tabular}




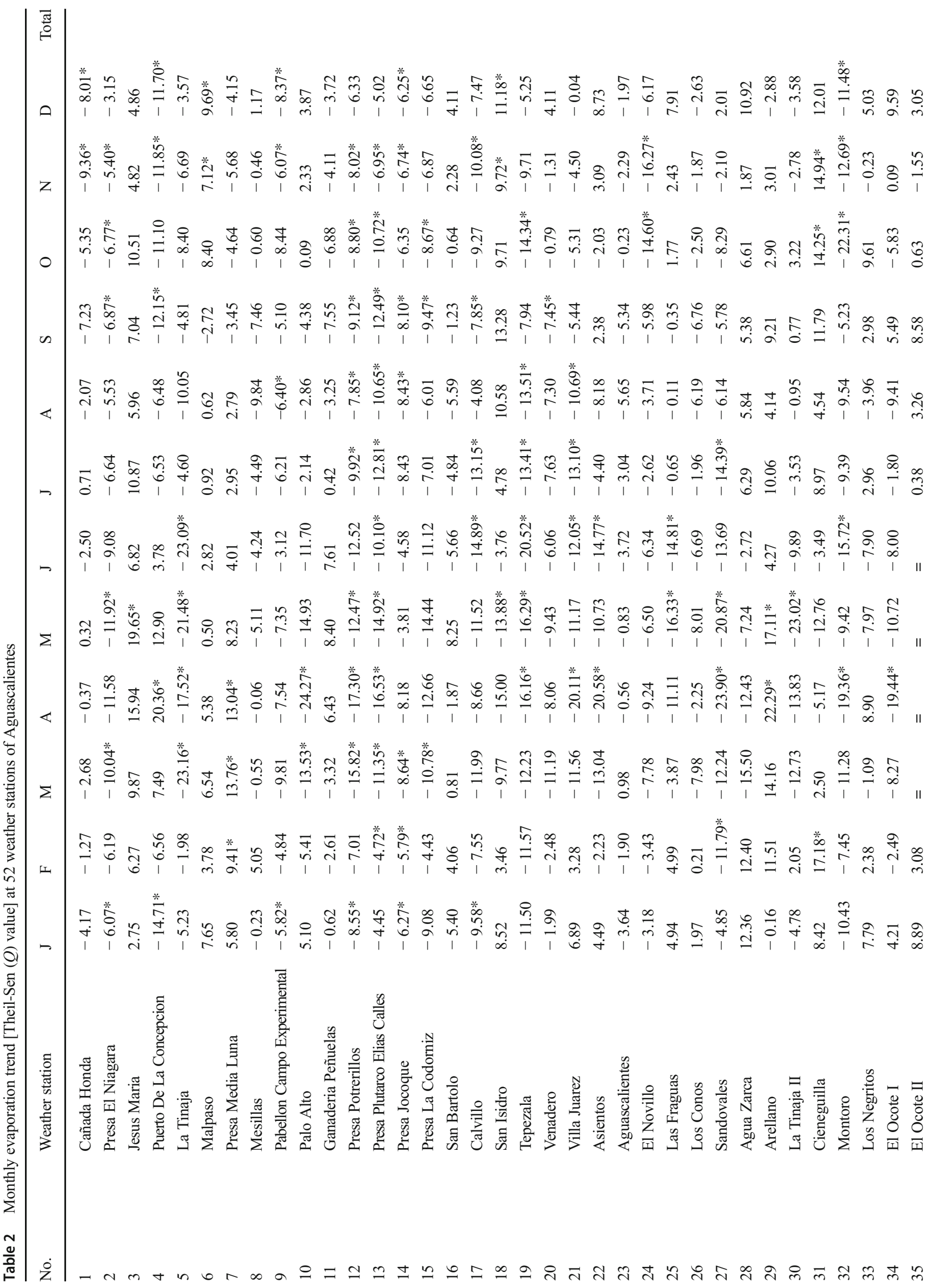




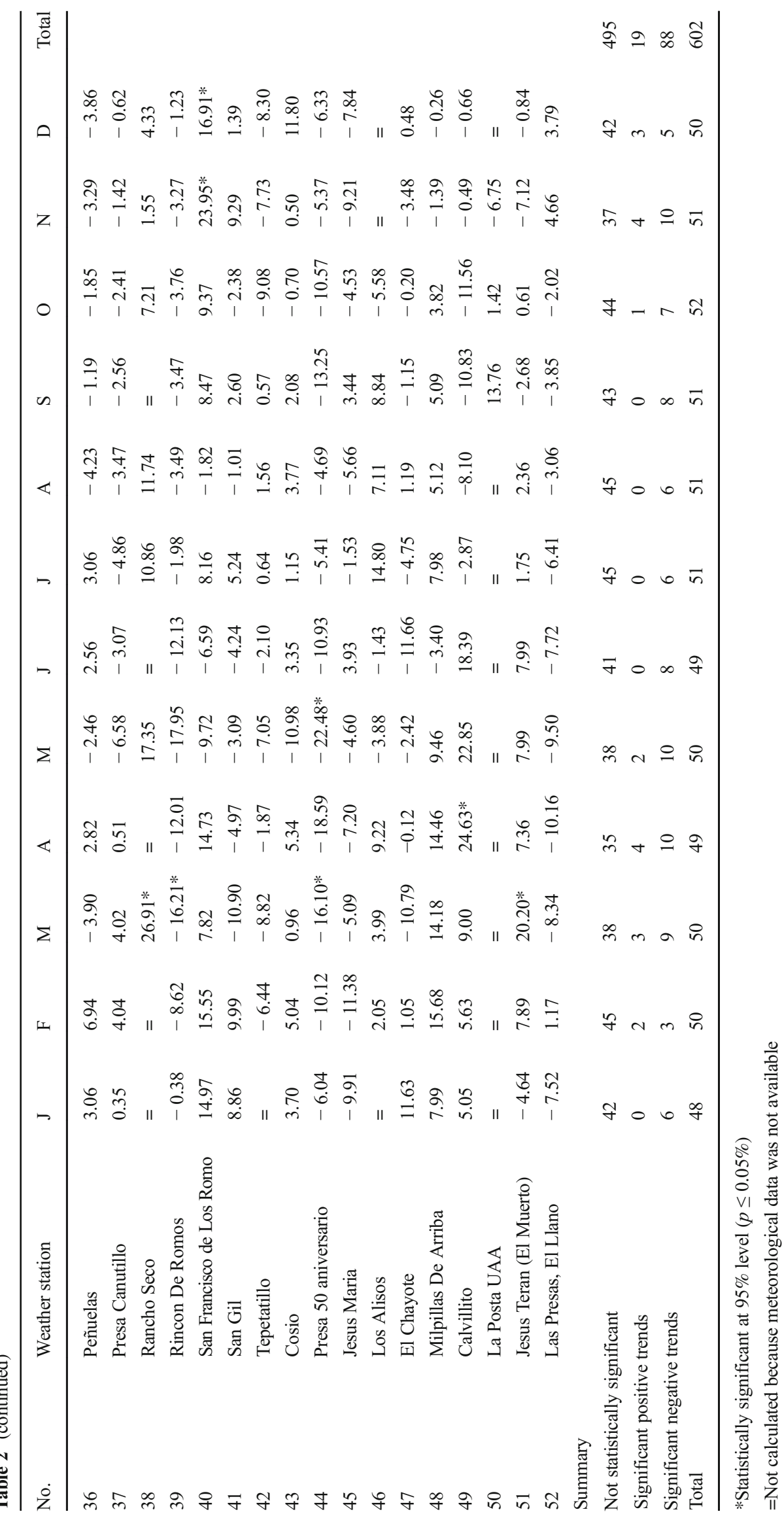

